\title{
Validating team communication data using a transmission-duration threshold and voice activity detection algorithm
}

\author{
Simon G. Hosking ${ }^{1} \cdot$ Christopher J. Best $^{1} \cdot$ Dawei Jia $^{1} \cdot$ Peter Ross $^{1} \cdot$ Patrick Watkinson $^{2}$ \\ Published online: 12 November 2018 \\ (C) Her Majesty the Queen in Right of Australia 2018
}

\begin{abstract}
The processes underlying team effectiveness can be understood by analyzing the temporal dynamics of team communication sequences. The results of such analyses have shown that the complexity of team communication is associated with team performance on task-related variables, and hence communication complexity statistics have been proposed for use as measures for real-time feedback on team performance. In two analyses of historical team communication sequences, we found that filtering via use of a transmission-duration threshold and voice activity detection algorithm resulted in significant changes in complexity relative to not filtering the data or using a transmission-duration filter alone. The use of these filtering techniques showed significant effects on the complexity of communication sequences in both a laboratory-based experiment, with participants with little experience with voice communication protocols, and in a mission simulation with trained military operators. There was also a significant non-linear relationship between the complexity of communication sequences and task performance. However, an analysis of the impact of the changes in communication dynamics gained through filtering did not demonstrate that the changed temporal dynamics of filtered data better explained team performance. It is concluded that prefiltering of invalid communication data should be included during the data cleaning stage of statistical analysis as a matter of good scientific practice. Furthermore, such use of filtering will ensure that inferences made about the relationship between the complexity of communication between team members and their performance are not confounded by the presence of invalid communication events.
\end{abstract}

Keywords Team communication $\cdot$ Sample entropy $\cdot$ Communication sequence filtering

\section{Introduction}

The importance of team effectiveness has been recognized in many safety-critical work settings such as military command and control, medical teams, and emergency services. Numerous studies have attempted to identify the processes underlying team effectiveness, as well as examine the efficacy of interventions designed to improve such processes (Kozlowski \& Ilgen, 2006). Many of those have established that communication is vital for the interaction

Simon G. Hosking

simon.hosking@dst.defence.gov.au

1 Department of Defence, Aerospace Division, Defence Science and Technology, 506 Lorimer Street, Port Melbourne, VIC, 3207, Australia

2 Department of Psychology, Deakin University, 221 Burwood Highway, Burwood, 3125, VIC, Australia and coordination of teams (Cooke et al., 2012a, b, 2013). Communication between two or more team members interacting to achieve a common goal is an observable behavior that changes over time according to the demands of a task. Over the last 20 years or so, it has been shown that understanding the temporal characteristics of team communication can be achieved using non-linear dynamics (e.g., Gorman et al., 2012; Guastello 1998a, b, 2000; Murase et al., 2017; Russell et al., 2012; Parker et al., 2016).

One non-linear approach to understanding team communication that has garnered recent momentum has been to measure the complexity of nominal and ordered communication sequences. ${ }^{1}$ These communication data are records of the sequential order of communication utterances for

\footnotetext{
${ }^{1}$ We note that there are many dynamical systems analyses that do not focus on regularity/complexity. For example, the work of Guastello and colleagues (Guastello 1998a, b, 2000) aimed to understand the patterns in communication data sequences that were robust and stable (see also Murase et al., 2017).
} 
all team members over time, with each team member represented nominally. An example of such data for a team of three speakers is shown in Fig. 2. The complexity of these communication sequences measured, for example, using sample entropy (see Richman \& Moorman 2000) or percent determinism (\%DET) (obtained from recurrence quantification analyses, RQA; see Coco \& Dale 2014), have been shown to vary according to team performance. For example, Gorman et al. (2012) measured the percent determinism of nominally discrete communication sequences using RQA and found that team communication sequences became more regular over time for teams that remained intact relative to those where the composition of the team changed over the course of the experiment. Given that mixed teams performed better on task-performance measures, Gorman et al. (2012) concluded that relatively greater complexity is symptomatic of more flexible and adaptable teams. Similarly, Gontar et al. (2017) used RQA to demonstrate that recurrence rates (in this case, a measure of the coupling of communication between flight captain and first officer) of flight-crew dyads could be successfully used to discriminate between high-performing and low-performing teams. Strang et al. (2012) applied nonlinear analyses to sequential team verbal communications recorded during a dyadic Air Battle Management (ABM) simulation. They found that for data coded for semantic content, decreases in sample entropy (i.e., a statistical measure of the amount of complexity/determinism in a system; see below) were associated with teams exposed to high workload. These changes in sample entropy have subsequently been shown to be dependent on the number of semantic categories in communication data sets, either through the scheme underlying the categorization process and/or the interaction between number of speakers and semantic content (Parker et al., 2016). Recently, Wiltshire et al. (2018) also measured the complexity of ordered nominal communication sequences recorded from dyads working on a complex problem solving task (i.e., the NASA Moonbase Alpha Simulation). For each recording, they calculated the entropy for sliding windows of communication sequences, and found that peaks in entropy were associated with problem solving phase transitions. That is, by tracking the level of entropy of communication sequences over time, they were able observe transitions in team's collaborative processes that can be categorized into the production and adaptation phases described in Ishak and Ballards' (2012) nested phase model for teams.

One of the advantages of applying non-linear methods to team communication data is that complexity measures could provide near real-time information about team performance without recourse to semantic analysis (e.g., Gorman et al., 2012). Non-linear analyses of team communication sequences could potentially be used for real-time feedback on team performance, or provide a source of objective data to be used for communication of operator states between human and/or synthetic teams (e.g., Cooke et al., 2013). However, if the results of these analyses are to be used successfully, then the validity of the data within each data sequence should be free of invalid communication signals such as extraneous noise. We defined the noise in communication data as invalid communication data that occurred when a speaker has pressed the push-to-talk but has not spoken, and can result from both unintentional and intentional but subsequently aborted communication events. For example, we have observed from our data instances where push-to-talk events did not have any speech content, and/or were so brief (i.e., communication events less than approximately $200 \mathrm{~ms}$ ) that the operator either involuntarily and briefly triggered the push-to-talk foot pedal without communicating, or aborted the communication to avoid "stepping on" (i.e., interrupting) a communication that was still in progress. In the physiological and kinematic fields, filtering of data to eliminate noise is common practice via the use of band-pass filtering or signal-confidence ratings (e.g., Weippert et al., 2014). To our knowledge, however, previous studies of the complexity of team communication sequences have not reported whether pre-filtering of invalid communications was carried out.

One potential method for filtering communication sequences is to have a human listen to each utterance prior to analysis to ensure that it is valid. However, such an approach is time-consuming and costly, and near-realtime analysis of communication complexity would not be possible. We investigated two alternative techniques for automatically filtering out invalid communication data. Although we were specifically interested in filtering invalid data from recordings of communication events using a push-to-talk system, any communication recordings that have been discretized before further analysis would benefit from the approach described below. For the first method, we used a minimum duration threshold filter on the basis that any communication duration less than threshold was unlikely to contain a valid utterance. For the second method, we used a Voice Activity Detector (VAD) algorithm (viz, Adaptive Multi-Rate Wideband; ARM-WB; see Bessette et al., 2002) to check each communication for audio content. The overall aims of this study were to (i) determine the frequency of invalid communications events between team members that occurred in either a highly controlled laboratory based experiment with relatively inexperienced communicators, and in a complex high-fidelity simulation exercise with highly experienced military operators; (ii) 


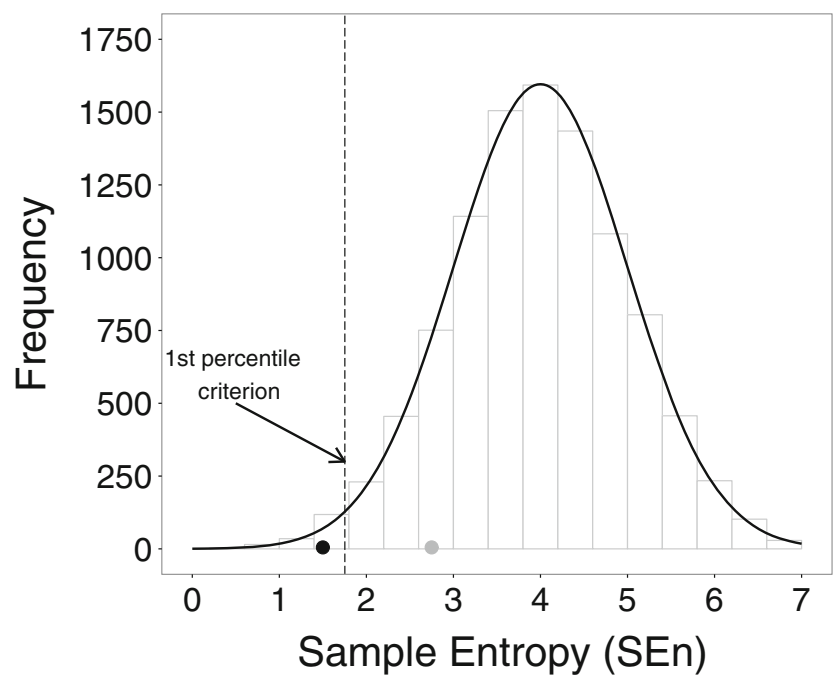

Fig. 1 Example histogram of 10,000 surrogate SEn values with a normal distribution curve (solid black line). The dashed vertical line shows the 1st percentile criterion for rejecting the null hypothesis. The gray dot-point denotes a hypothetical SEn value obtained from an original communication sequence prior to filtering. The black dotpoint denotes a new SEn value obtained from the same communication sequence post filtering. Note that, in this example, the SEn value prior to filtering the communication sequence is not significantly different to stochastic noise; however, filtering the communication sequence results in the new SEn value being significantly different

determine the potential for invalid communication events to influence the results of complexity analyses, and (iii) determine whether the filtering of invalid communication events from communication sequences had any effect on the relationships between the complexity of communication and task performance.

\section{General methods and analyses}

The analyses reported in this study were performed on historical data obtained from (i) a laboratory-based team experiment carried out in fulfillment of a Masters thesis (Watkinson et al., 2015), and (ii) an Air Battle Management simulation exercise (Francis et al., 2015) carried out as part of a series of biannual simulation exercises used for research and training within the Royal Australian Air Force (RAAF). Neither of these studies have previously had their communication data analyzed using the techniques described below. We briefly describe the methods used in each study below; a more detailed description can be found in Watkinson et al. (2015) and Francis et al. (2015), respectively. Communications between team members in both the laboratory-based experiment and simulation exercise were transmitted using a push-to-talk communication system that was compliant with the Distributed Interactive Simulation (DIS) protocol. DIS uses two mechanisms for publishing communication events to a network. When an operator engages the pushto-talk pedal and speaks into a microphone, a Transmitter State Protocol Data Unit (PDU) is sent to indicate that the radio is transmitting. Following this PDU, Signal PDUs containing buffers of mu-law compressed audio at regular discrete time intervals are transmitted. On release of the push-to-talk button, the simulator sends another Transmitter State PDU indicating that the radio is no longer transmitting. Hence, the total transmit duration of the communication events are calculated by comparing the times at which the Transmitter PDUs were sent and summing the signal PDU buffers.

In order to investigate the effects of different types of filtering on communication data, three filtering conditions were analyzed: (i) a condition in which no filter was used on the data, (ii) a second condition in which data were filtered on the basis of a minimum communication duration-threshold (time-only), and (iii) a third condition in which data were filtered using a combination of the time-only filter and a voice activity detector (VAD) algorithm (time+VAD; see below). The data were filtered using software that carried out the above two filtering requirements and flagged invalid communications. The communication duration-threshold filter excluded communication events that were too short to contain meaningful information. We based this threshold on the results of a pilot experiment in which we measured the minimum time-to-transmit monosyllabic words using the same pushto-talk, DIS-based, radio used in the laboratory experiment and simulation exercise described below. The results from five participants indicated that the minimum time needed to transmit (i.e., the overall minimum time to transmit a single word based on five participants communicating 25 words repeated 5 times for a total of 625 words) was $220 \mathrm{~ms}$.

The VAD algorithm described in the AMR-WB audio compression standard was used to measure the proportion of each communication event where the signal PDU contained voice activity. The VAD algorithm examined the energy levels within each communication event at different frequencies by splitting the event into 20-ms bins and then determining whether each bin contained sufficient data for transmitting (encoding). The system split the input waveform into 12 bands ranging from 0 $200 \mathrm{~Hz}$ (band 1) to $4800-6400 \mathrm{~Hz}$ (band 12). For each band, the algorithm performed a signal-to-noise (SNR) calculation based on historical noise estimation. The 12 SNR values were then summed together. A Boolean is 
returned depending on whether the summed value is greater than a threshold. The threshold is automatically updated based on measures of background noise and signal level. A more detailed description of VAD is available in Bessette et al. (2002). The software then counted the number of VAD true frames within a single communication event and these were recorded as a proportion of total communication duration. An algorithm of the programming code used to implement the VAD and duration-threshold filtering techniques is provided in Algorithm 1. This proportion was then multiplied by the communication duration such that communication events were excluded if the product of the communication duration and VAD proportion was less than the duration-threshold (i.e., $220 \mathrm{~ms}$ ).

$\overline{\text { Algorithm } 1 \text { The following pseudo-code has been written }}$ to show, in general form, how the time-threshold and VAD filters can be implemented. While it has been written to represent the programming code that we used for the filtering of DIS PDUs, it could equally be applied to any programming language and for other audio file formats that have been discretized. Given an input file containing DIS PDUs, for each communication event, the code outputs the timestamp, duration and VAD proportion

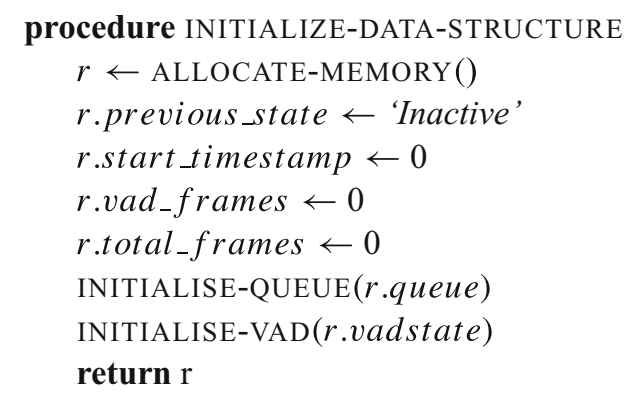

procedure PROCESS-TRANSMITTER-PDU(D, pdu, timestamp)

$i d \leftarrow$ (pdu.entity_identifier, pdu.radio_identifier)

if $i d \notin D$ then

$D[i d] \leftarrow$ INITIALISE-DATA-STRUCTURE ()

$r \leftarrow D[i d]$

if r.previous_state $\neq$ 'Transmitting' and pdu.state $=$ 'Transmitting' then

r.start_timestamp $=$ timestamp

r.vad frames $\leftarrow 0$

r.total frames $\leftarrow 0$

INITALISE-QUEUE(r.queue)

INITIALISE-VAD(r.vadstate)

else if $r$.previous state $=$ 'Transmitting' and pdu.state $\neq$ 'Transmitting' then

duration $\leftarrow$ timestamp - r.start_timestamp

if $r_{\text {.total_frames }}>0$ then score $\leftarrow$ r.vad_frames $/$ r.total_frames else

score $\leftarrow 0$

vad_proportion $\leftarrow$ duration $*$ score

OUTPUT-COMMUNICATION-EVENT(id,

r.start_timestamp, duration, vad_prop-

ortion)

r.previous_state $\leftarrow$ pdu.state

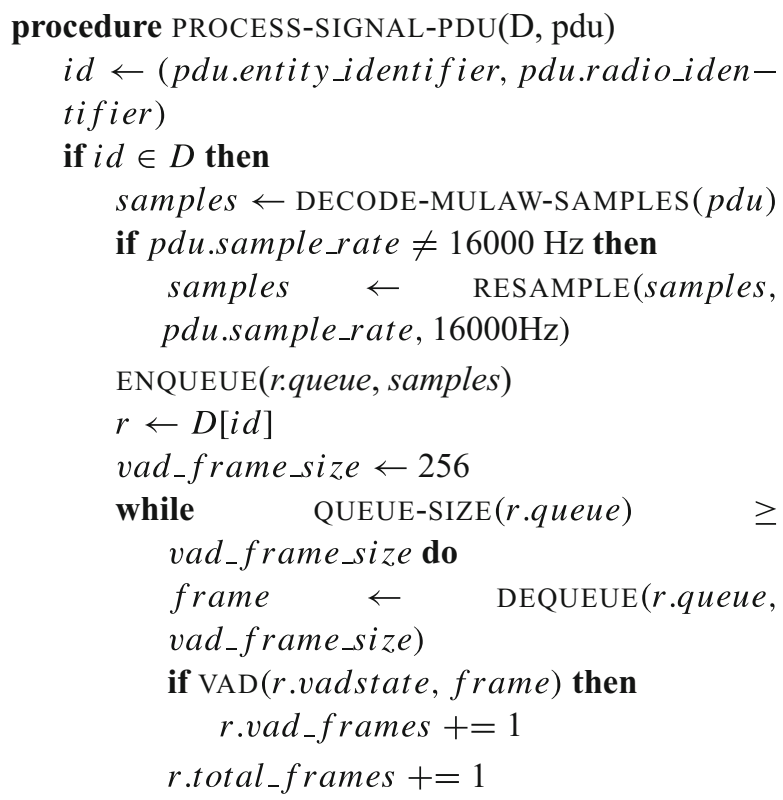

procedure PROCESS-DIS-LOG-FILE(file) $D=\{\}$

while END-OF-FILE $($ file $) \neq$ true do

(pdu, timestamp $) \leftarrow$ READ-DIS-PDU $($ file $)$

if pdu.type $=$ 'Transmitter PDU' then

PROCESS-TRANSMITTER-PDU $(D, p d u$, timestamp)

else if $p d u . t y p e=$ 'Signal PDU' then

PROCESS-SIGNAL-PDU $(D, p d u)$

Communication sequences were coded numerically, with each number representing one of each of the team members. The time and duration of each communication was also recorded. These sequences were then subjected to Sample Entropy (SEn) analyses. SEn is the negative natural logarithm of the conditional probability that patterns in a sequence of data are repeated (Richman \& Moorman, 2000). A lower level of SEn reflects less complexity in the data sequence, and conversely, increased regularity. While, as pointed out above, there are numerous non-linear statistics available to determine the temporal dynamics of nominal communication sequences (e.g., output measures from CRQA; approximate entropy [AppEn]; Kolmogorov-Sinai 
entropy) we chose to use SEn over (i) other forms of entropy statistics as it is more suited to small data sets (see below) and less influenced by noise (Yentes et al., 2013), and (ii) other measures of complexity/determinism available from CRQA due to SEn involving parameter selection that is less subjective than those used in CRQA. SEn uses three parameter settings ( $r$, tolerance value; $m$, template pattern length; and $n$, sequence length) and selection of these are relatively straightforward, especially for nominal data sequences. For the following analyses, we set $r=.00001$ (approximating zero tolerance due the fact that the data are not continuous time-series) and $m=2$ with $n$ varying depending on the level of analysis required. It has been shown that SEn is largely independent of $n$, and is consistent over various parameter settings (e.g., Richman \& Moorman, 2000; Yentes et al., 2013). However, the robustness of SEn is dependent on a sequence length of at least $n=80$ (Sokunbi, 2014).

For the analyses described below, comparisons were made between obtained SEn values (for each communication sequence and each of the three filtering conditions) and the 1 st percentile of their surrogate distribution. Such a comparison is typically carried out to ensure that nonstochastic temporal structure (as opposed to stochastic noise) exists in the data sequences prior to analysis. Surrogate data testing is a technique similar to parametric bootstrapping, and for our analyses it comprised of randomly shuffling each original communication sequence, and then determining a SEn value for the resultant shuffled sequence. This process is repeated $n$ times (the following analyses used $n=10,000$ ) such that a distribution of SEn values is obtained. Due to the rule of large numbers, these multiple repetitions result in a distribution of surrogate SEn values that converges on a normal distribution with a stable mean and variance (i.e., $N\left(\mu, \sigma^{2}\right)$. The mean of the surrogate distribution therefore reflects the amount of SEn that would be expected due to chance. A null hypothesis test was then carried out by comparing the obtained SEn value from the original communication sequence to the 1st percentile of the surrogate distribution (see Fig. 1). If the obtained SEn was less then the 1st percentile of the surrogate distribution, then it was concluded that the temporal structure of the original communication is significantly different to that which would be expected due to chance occurrence. Hence, it should be noted that when we refer to low and high entropy, these are relative comparisons with the caveat that high entropy should still be significantly less than that expected by chance (which would reflect a system behaving randomly rather than one which is relatively high in complexity; c.f., Wiltshire et al., 2018). We compared the effects of filtering on these surrogate analyses by counting the number occasions in which the obtained SEn values changed from being less than the 1st percentile for unfiltered sequences to greater than the 1st percentile for filtered sequences, and vice versa. For convenience, these events have been termed reversals, and an example of such a reversal is shown in Fig. 1.

\section{Analysis of laboratory-based team communications}

\section{Method}

We first measured the occurrence and effect of invalid communication data that were collected from the laboratorybased team performance experiment. The experiment consisted of ten teams of three participants. Participants in each team were randomly allocated to one of the three roles, with each role necessary to complete the task of seeking-and-destroying a single simulated enemy aircraft embedded in a wave of unidentified aircraft that traversed a computer display, while simultaneously defending various assets. Each wave of aircraft appeared at approximately 60$\mathrm{s}$ intervals, and disappeared following a successful attack on the target aircraft. All aircraft were under the control of the Distributed Dynamic Decision-making (DDD) simulation software (MacMillan et al., 2004) that simulated air battle surveillance and management. Each team was required to complete 24 simulated missions over a 4 -week period, each mission taking approximately $20 \mathrm{~min}$ to complete. In order to complete the task, team-members had to work together and coordinate their behavior by transmitting communications via push-to-talk radios about the status of the attacking

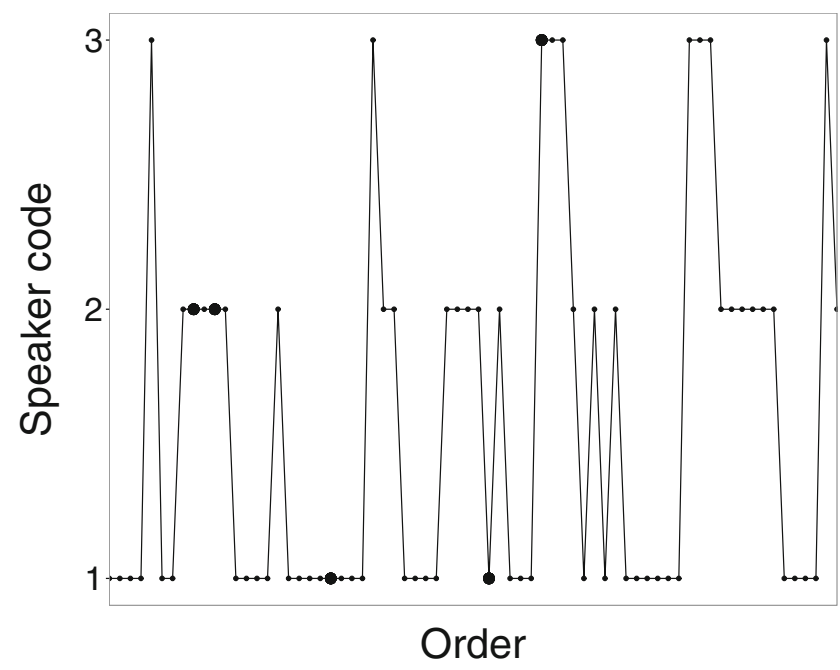

Fig. 2 Example sequence of ordered speaker communications for the laboratory-based experiment data. The smaller filled circles show the speaker sequence. The larger filled circles show invalid communications identified by a time+VAD filter (see the General methods and analyses section for details) 
wave, and the location of a target aircraft. Figure 2 shows an example communication sequence of one of the teams over a single mission. Data from one team were discarded because the recorded sequence lengths from that team were considerably less than the $n=80$ minimum. Hence, the analyses were completed on a total of 216 communication sequences ( 9 teams $\times 12$ tasks $\times 2$ repetitions).

\section{Results}

The presence of invalid communication events was evidenced by a reduction in the length of the communication sequences as a function of the two different filter conditions (i.e., the time-only and time+VAD, filters). A total of $191(88.4 \%)$ in the time-only filter condition, and 211 $(97.7 \%)$ in the time+VAD filter condition of all communication sequences had at least one invalid communication. The mean difference in sequence length between the unfiltered sequences and filtered sequences were 4.61 (2.3\%) and 8.11 (4.5\%) for the time-only, and time+VAD filtering, respectively. Figure 3 shows the differences in sequence lengths for time-only filtering, and time+VAD filtering, as a function of the original, unfiltered length of each sequence. An analysis of the relationship between the unfiltered sequence length and the filtered length was carried out on a subset of the data where the difference between the filtered and unfiltered data lengths were greater than zero; this was because the change in sequence-length data was not normally distributed and required log-transformations. A linear mixed-effects model on the log of the length differences (with Kenward-Roger approximation for degrees of freedom) showed a significant linear relationship between differences in sequence lengths and original sequence

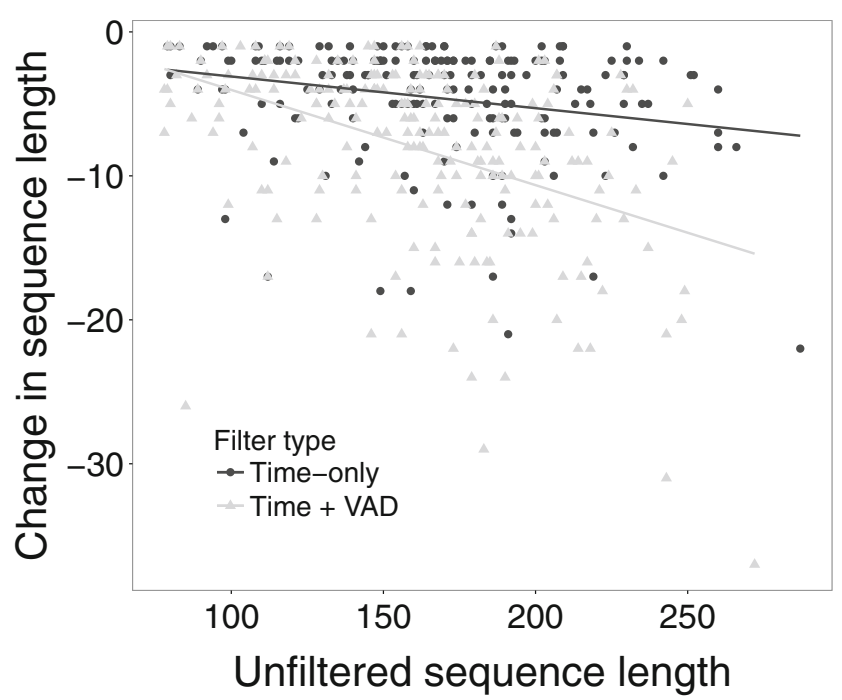

Fig. 3 Change in filtered sequence lengths as a function of unfiltered sequence length for time-only, and time+VAD filtering length $F(1,397.95)=69.165, p<.0001$, and a significant interaction between sequence length and filter type $F(1,390.16)=6.97, p<.01$. Overall, the regression model accounted for $41.5 \%$ of the variance in changes to sequence length. As can be seen in Fig. 3, longer sequence lengths typically had larger changes due to filtering, indicating that the longer the communication sequence in a session, the greater the number of invalid communications. Across all unfiltered sequence lengths, the number of invalid communications using the time-only filter were approximately half that of the time+VAD filter.

The analyses of the relationship between different filter types and associated changes in the SEn of communication sequences was performed at two different levels of comparison. First, we compared the SEn values of the unfiltered communication sequences with those of the filtered sequences. with the length of each unfiltered sequence trimmed to the length of its matching filtered sequence. This level of comparison was designed to show the effect of filtering on SEn values where there were a large and variable number of both filtered and unfiltered sequence lengths, therefore increasing the generalizability of the results. Second, the same comparison was made but with the length of the unfiltered sequence trimmed to match that of the shortest filtered sequence across both filtering conditions, such that all communication sequences were identical in length. Only a subset of data was used where there was a change in sequence length of at least one event. Each sequence was analyzed to produce a SEn statistic pre-and-post filtering. As described in the General methods and analyses section, the obtained SEn values in each condition were also compared to the first percentile of the surrogate data to determine whether the obtained SEn values were significantly different to those that would be expected due to chance. All linear regression analyses of the relationship between SEn and filtering were conducted using a linear mixed-effects model with the difference in SEn values and the type of filter used treated as fixed effects, and team treated as a random effect. Since the regression models were multi-level, fixed effects were approximated using Kenward-Roger approximations.

Figure 4 shows that when unfiltered sequence lengths were trimmed to that of their matching filtered lengths, differences in SEn values were significantly associated with the original length of the unfiltered sequence $F(1,419.01)=18.40, p<.001$ and the type of filter used $F(1,411.09)=21.85, p<.001 ; R^{2}=.336$. Differences in SEn values for filtered data relative to the unfiltered data (holding filter type constant) were relatively larger for shorter unfiltered sequence lengths, and these differences decreased linearly as sequence length increased. Furthermore, differences in SEn values for the time+VAD filter were larger than those produced for the time-only filtered 


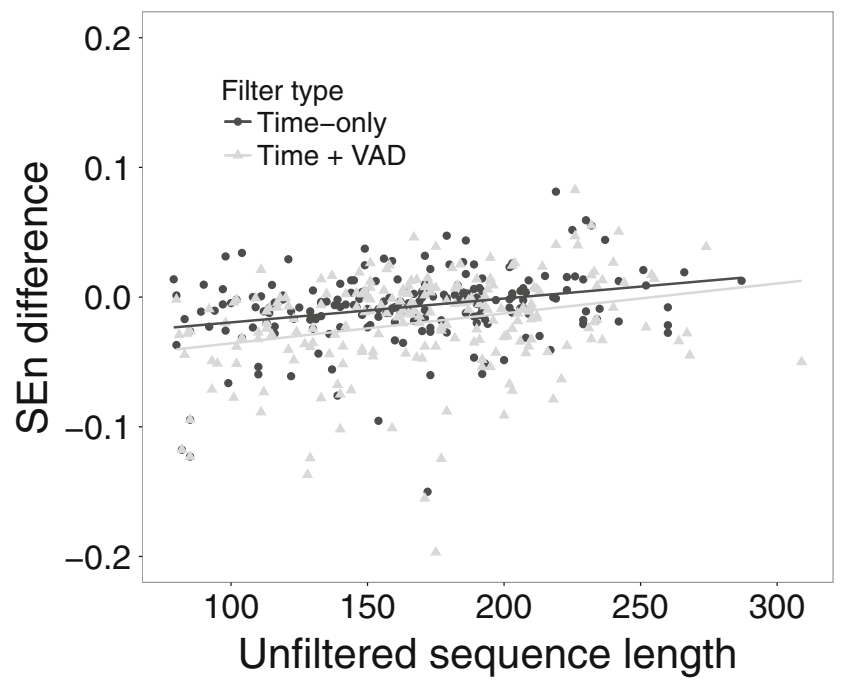

Fig. 4 Difference in SEn values for communication sequences as a function of unfiltered sequence length and filter type, where the length of the unfiltered sequence was trimmed to the length of its matching filtered sequence

data. Post hoc comparisons, using Bonferroni corrections, also found that the differences between the mean SEn difference for the time-only filtered condition $(p<.01)$, and time+VAD filtered condition $(p<.001)$ data were significantly different from zero. Figure 5 shows that when unfiltered sequence lengths were trimmed to the minimum sequence length of the data set, SEn differences were significantly associated with the length of the unfiltered sequence $F(1,414.99)=10.58, p<.01$ and filter type $F(1,421.09)=8.98, p<.01 ; R^{2}=.179$. The relationship between differences in SEn and the length of the unfiltered sequences found for sequences trimmed to

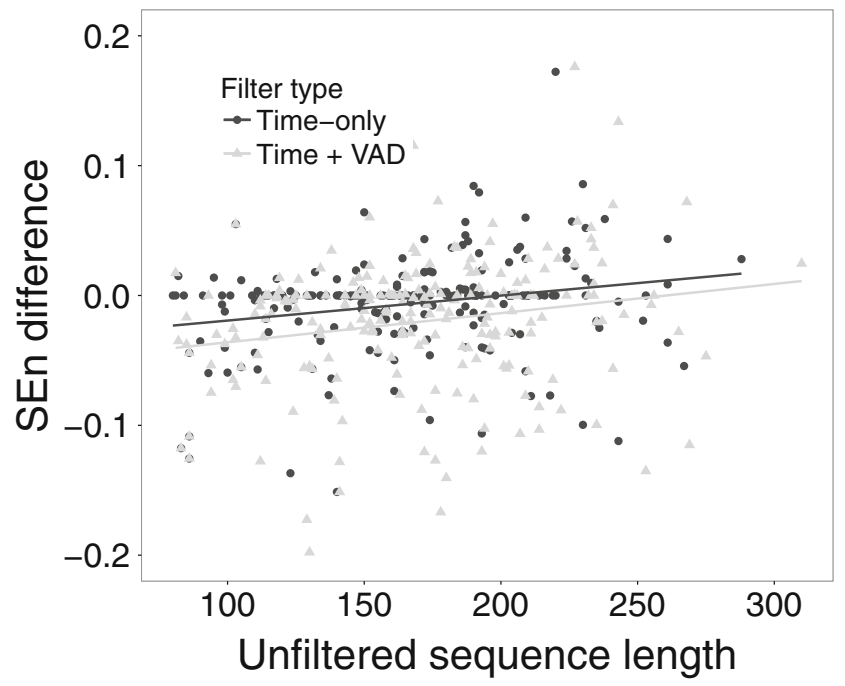

Fig. 5 Difference in SEn values as a function of unfiltered sequence length and filter type, where the length of all communication sequences were trimmed to that of the shortest overall sequence the minimum sequence length was very similar to that shown in Fig. 4 for the matched sequence length data; that is, a larger difference for shorter sequence lengths, and a linear decrease in SEn differences as the sequence lengths increased. Also consistent were the larger SEn differences for the time+VAD filter relative to the time-only filter. Post hoc comparisons, using Bonferroni corrections, between the mean SEn for time-only $(p<.001)$, and time+VAD $(p<.001)$ filtered data were also significantly different to that of the no filter condition. As can be seen in Table 1, filtering the communication sequences also resulted in a greater number of reversals in which the obtained SEn values changed from being less than the 1st percentile when unfiltered, to greater than the 1st percentile following time-only or time+VAD filtering. Furthermore, there were a greater number of reversals in this direction for the time+VAD filtered data compared to that of the time-only filtered data.

\section{Analyses of mission simulation communications}

The analyses presented above have shown that invalid communications were present in team communication data and had a significant influence on estimates of the complexity of communication sequences. These results suggest that communication sequences should be filtered prior to analysis. One of the problems of injecting filtering algorithms to communication data is that it may produce lag in a system designed to produce real-time analysis and feedback of team performance. Therefore, it would be preferable if filters were not present in real-time data analyses. The above analyses were carried out in a highly controlled experimental setting with participants whom were not formally trained in communication protocols. Hence, it is possible that invalid radio communications are less problematic in applied settings where speakers are highly trained to effectively and efficiently use push-to-talk communication systems and avoid invalid communications. In order to check the generalizability of the above analyses and affirm the need to apply communication filters to realtime systems, we next investigated the presence of invalid communication data and their effect on SEn values for recorded communications between team members in an ABM simulation training exercise, where speakers were military operators that were highly experienced at radio communication.

\section{Method}

Ten collaborating operators participated in the simulated missions, including an air battle director, tactical director, 
Table 1 Frequency of unfiltered communication sequences that had SEn values that reversed from being greater than first percentile $\Phi_{01}$ of surrogate distribution to less than first percentile $\left(\operatorname{SEn}<\Phi_{01}\right)$, or from being less than first percentile to greater than first percentile (SEn $>\Phi_{01}$ ) following either time-only or time+VAD filtering

\begin{tabular}{lllll}
\hline & \multicolumn{2}{c}{ Time-only } & & \multicolumn{2}{c}{ Time+VAD } \\
\cline { 2 - 3 } & SEn $<\Phi_{01}$ & SEn $>\Phi_{01}$ & & SEn $<\Phi_{01}$ \\
\hline Matched length $^{\mathrm{a}}$ & 2 & 15 & 4 & 22 \\
Minimum length $^{\mathrm{b}}$ & 3 & 11 & 4 & 19 \\
\hline
\end{tabular}

${ }^{a}$ Reversal frequencies obtained when the length of the unfiltered sequences were trimmed to the length of its matching filtered sequence

${ }^{\mathrm{b}}$ Reversal frequencies obtained when the length of all communication sequences were trimmed to minimum length of the filtered sequences

fighter controllers, mission commander, and surveillance and control operators. A total of seven unique and loosely scripted exercise scenarios (each lasting $60 \mathrm{~min}$ ) were carried out over 4 consecutive days. Communication data were coded nominally (and then transferred into numerical codes) for each speaker and each scenario (see Fig. 6), along with the time and duration of each utterance. The number of participating operators who spoke during each mission varied from 8 to 10 . SEn values were derived from moving windows of 80 utterances, with a step size of $10 \%$ of the window size (i.e., eight utterances). These window sizes closely approximated the lengths of sequences which were used as the minimum sequence length in the laboratory data analyses. As in the previous analyses, SEn values from communication sequences were compared pre-andpost filtering, where post filtering consisted of time-only or time+VAD filtering.

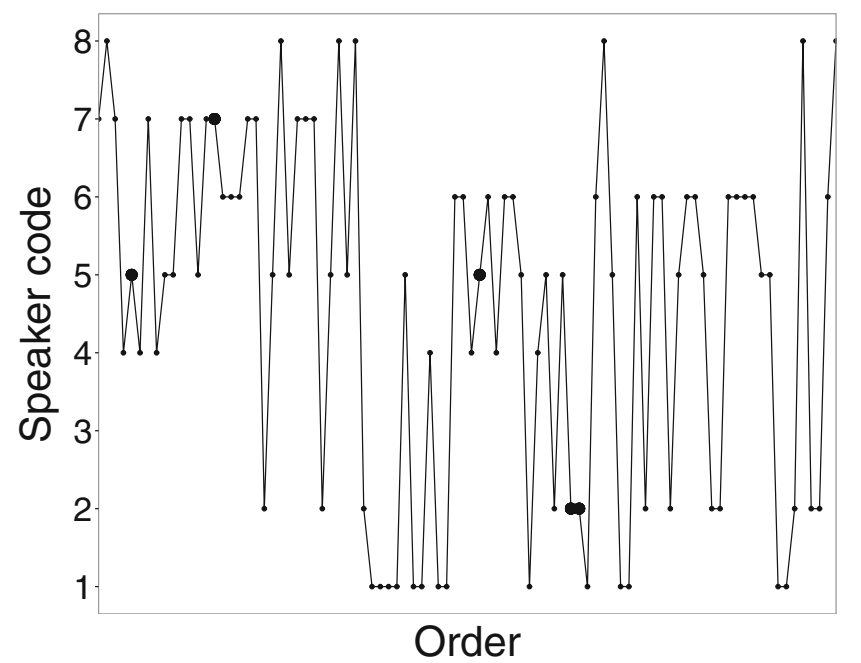

Fig. 6 Example sequence of communications of ordered speaker codes for the ABM simulation data. The smaller filled circles show the speaker order and code. The larger filled circles show invalid communications (as determined by a time+VAD filter; see text for details)

\section{Results}

Table 2 shows the changes in sequence lengths of the simulation data that occurred as a function of filtering invalid communications using the time-only, or time+VAD filter. The mean change in sequence lengths relative to the unfiltered sequence length for the time-only filter was approximately half that of the time+VAD filter. For each communication sequence window, we calculated surrogate data using the same method as that described above. We then compared the SEn value obtained in each window to the 1st percentile of its surrogate distribution. An example time series of SEn values with the 1st percentile of the surrogate distribution for one window is shown in Fig. 7. As can be seen in Fig. 7, some of the obtained SEn values were less than the 1st percentile of the surrogate distribution, indicating that for these windows of communication data there was significantly less complexity in team communications than that expected by chance occurrence. As can be seen in Table 3, the filtering of these data resulted in a number of reversals of this result such that SEn values for unfiltered data windows changed from being less than the first percentile to greater than the first percentile following filtering, and vice versa (see e.g., the shaded boxes A and B in Fig. 7). Overall, as can be seen from the median statistics in Table 3, there did not appear to be any systematic differences in the magnitude and/or direction of the reversals, with the exception that the time+VAD filter produced more reversals than that of the time-only filter.

In order to determine how the filtering of data changed the overall pattern of SEn values relative to those of the unfiltered data, we carried out cross-correlations of SEn time series for the unfiltered and filtered data for each of the seven missions. The cross-correlation of the SEn series measured the similarity between the unfiltered SEn series and shifted (lagged) copies of the filtered sequence as a function of the lag. An example graphical output of the cross correlation analysis of the 7th mission simulation 
Table 2 Summary statistics for unfiltered, time-only filtered, and time+VAD filtered, data sequences

\begin{tabular}{|c|c|c|c|c|c|}
\hline & \multicolumn{3}{|c|}{ Sequence length } & \multicolumn{2}{|c|}{ Percent invalid } \\
\hline & Unfiltered & Time-only & Time+VAD & Time-only & Time+VAD \\
\hline Scenario 1 & 760 & 738 & 721 & 2.9 & 5.1 \\
\hline Scenario 2 & 1058 & 1036 & 1014 & 2.1 & 4.2 \\
\hline Scenario 3 & 1021 & 998 & 987 & 2.3 & 3.3 \\
\hline Scenario 4 & 1089 & 1064 & 1046 & 2.3 & 3.9 \\
\hline Scenario 5 & 898 & 876 & 856 & 2.4 & 4.7 \\
\hline Scenario 6 & 1260 & 1219 & 1201 & 3.3 & 4.7 \\
\hline Scenario 7 & 1240 & 1220 & 1202 & 1.6 & 3.1 \\
\hline Mean & & & & 2.4 & 4.1 \\
\hline SD & & & & 0.5 & 0.8 \\
\hline
\end{tabular}

is shown in Fig. 8. Horizontal dotted lines show upper and lower $95 \%$ confidence intervals. Any cross-correlation above or below those confidence intervals was statistically significant. Table 4 shows that there was a lag between the maximum cross correlations (the peaks of the crosscorrelation lagged data) of the unfiltered data and that of the filtered data, and also between those of the time-only and time+VAD cross correlations. Moreover, Table 4 shows

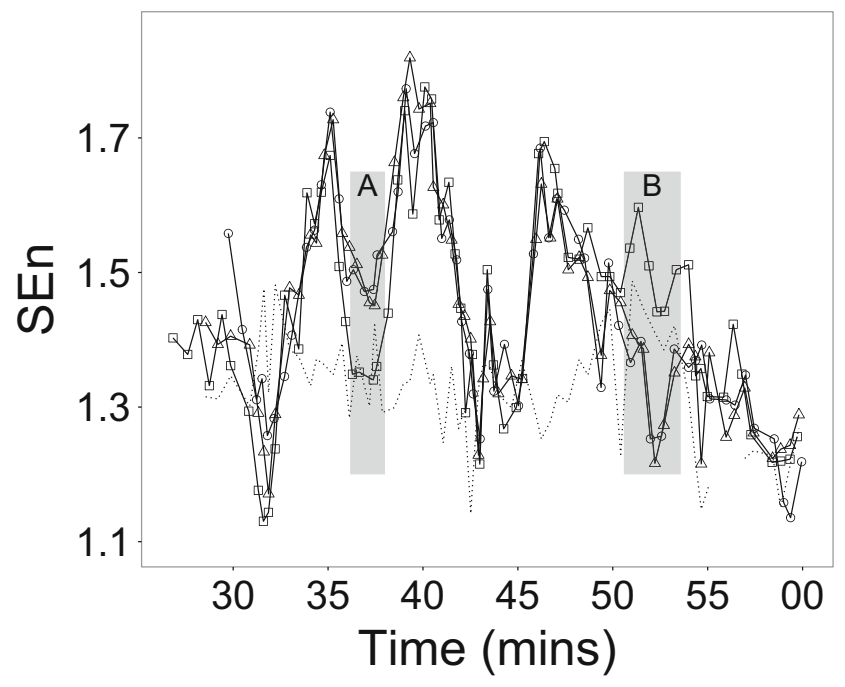

Fig. 7 Example time series plot of SEn values for each moving window over the simulated ABM mission for both unfiltered (open squares), time filtered (open triangles), and time+VAD filtered (open circles) data. The dotted line shows the 1st percentile of surrogate data generated within each window of SEn values. The gray-shaded boxes marked A and B provide examples of the reversals described in the text. Box A shows an example of the situation where the SEn from unfiltered data is significantly lower than that would be expected due to chance and the null hypothesis is rejected. Note that post filtering the null-hypothesis cannot be rejected. Box B shows the reverse case; that is, where the SEn from unfiltered data is not significantly different to that expected by chance, whereas post-filtering shows the reverse outcome that maximum significant cross-correlations coefficients ranged from 0.310 to 0.870 for the time+VAD series, with associated lags from 1-5 communication events, and from 0.525 to 0.940 for the time-only data with associated lags from 0-1 communication events. In summary, the cross correlations between the unfiltered and filtered data was always less than 1.0, with the statistically significant correlations between the unfiltered and filtered data varying from moderate to strong, and the cross-correlations of the filtered SEn data leading that of the unfiltered data.

\section{Analysis of effects of filtering on relationship between SEn and task performance}

Given that there is currently no consensus regarding optimum SEn values for specific tasks in absolute terms (Stergiou et al., 2006), relative differences between SEn values under different experimental conditions are used to indicate relationships between task performance and complexity. While the results of our analyses, reported above, have found highly significant relative differences in SEn values as a function of differing filter types, the magnitude of those differences were relatively small. In the following analysis, we investigated whether these relatively small differences in SEn that occurred due to filtering had an influence on the relationship between SEn and task performance in the laboratory task described above. SEn values derived from communication sequences were analyzed as a function of both filter type and the total number of hits that occurred during a single trial, where a hit was defined as a successful attack on the target aircraft on first attempt, for each wave of aircraft. Total hits per trial were stratified into four categories, with each category based on the 25 th, 50 th, and 75 th percentile, corresponding to : $<20,20-21,22-23$ and 24 hits per trial. We analyzed 
Table 3 Frequency of unfiltered communication sequences that had SEn values that reversed from being greater than first percentile $\Phi_{01}$ of the surrogate distribution to less than first percentile $\left(\mathrm{SEn}<\Phi_{01}\right)$ following filtering, or from less than first percentile to greater than first percentile $\left(\mathrm{SEn}>\Phi_{01}\right)$, as a function of time-only or time+VAD filtering

\begin{tabular}{lllll}
\hline & \multicolumn{2}{c}{ Time-only } & \multicolumn{2}{c}{ Time+VAD } \\
\cline { 2 - 3 } & SEn $<\Phi_{01}$ & SEn $>\Phi_{01}$ & SEn $<\Phi_{01}$ & 14 \\
Scenario 1 & 8 & 8 & 12 & 12 \\
Scenario 2 & 9 & 8 & 14 & 7 \\
Scenario 3 & 13 & 11 & 10 & 13 \\
Scenario 4 & 5 & 14 & 18 & 12 \\
Scenario 5 & 9 & 5 & 13 & 13 \\
Scenario 6 & 13 & 8 & 7 & 13 \\
Scenario 7 & 5 & 10 & 13 & 12 \\
Median & 9 & 8 & & 13 \\
\hline
\end{tabular}

these data using a mixed model ANOVA with SEn as the dependent variable, and filter type and total hits per trial as independent variables.

\section{Results}

Figure 9 shows the effects of filter type and number of hits per trial on the SEn values of communication sequences in the laboratory task. The ANOVA found significant main effects for both the total number of hits per trial $F(3,612)=$ $18.94 ; p<.001$ and filter type $F(2,10)=5.73 ; p<.05$.

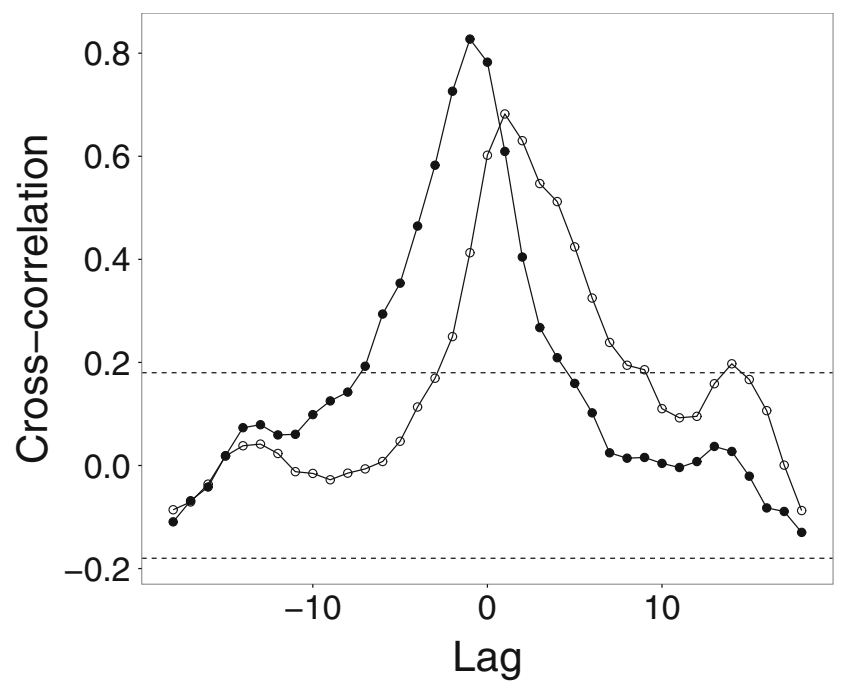

Fig. 8 Example cross-correlations between unfiltered and filtered SEn communication sequences. Dashed horizontal lines show $95 \%$ confidence intervals of cross-correlation coefficients. Open circles show cross-correlations between unfiltered and time+VAD filtered data; filled circles show cross-correlations between unfiltered and time-only filtered data
There was no significant interaction between filter type and total hits per trial. As can be seen in Fig. 9, as the number of hits in a trial increased (i.e., an increase in total number of successful attacks on first attempt), the complexity of communication sequences decreased. Post hoc comparisons with Holm corrections for Type-1 error (Holm, 1979) found that while there was no significant difference between the $<20$ and $20-21$ total hits categories, these two categories were significantly different to the $22-23$ hits per trial $(p<.001)$ and 24 hits per trial $(p<.001)$ categories. Note, however, that the reductions in SEn values as a function of total hits per trial was not monotonic. Rather, there was a significant increase in SEn between the $22-23$ hits and 24 hits categories ( $p=.012)$, resulting in a U-shaped function. Figure 9 also shows the main effect of filtering on SEn values. Consistent with the results of the effects of filtering on the complexity of laboratory-based and mission-based team communication sequences described above, post-hoc comparisons revealed that there were significant differences between all three levels of filtering types $(p<.001)$, with time+VAD filtering resulting in significantly larger SEn values $(\bar{X}=0.69$; se $=0.011)$ than the time-only $(\bar{X}=0.67 ; \mathrm{se}=0.011)$ and no-filtering $(\bar{X}=0.66$; se $=$ 0.012) conditions. However, it is clear from Fig. 9 that the effect of total number of successful hits per trial on the SEn of communication sequences was not influenced by the type of filtering used. The implications of these results will be discussed in the General Discussion.

\section{General Discussion}

One of the aims of this study was to measure the frequency of invalid communications in two separate teambased tasks. Duration threshold (time-only) filtering of 
Table 4 Maximum cross-correlations between unfiltered and filtered SEn time series, with lag in steps of a single voice communication event

\begin{tabular}{lllll}
\hline & \multicolumn{2}{l}{ Cor(unfiltered, time-only) } & & \multicolumn{2}{c}{ Cor(unfiltered, time+VAD) } \\
\cline { 2 - 3 } & Max-correlation & Lag (steps) & Max-correlation & Lag (steps) \\
\hline Scenario 1 & 0.822 & 1 & 0.740 & 3 \\
Scenario 2 & 0.774 & 1 & 0.727 & 4 \\
Scenario 3 & 0.913 & 1 & 0.790 & 1 \\
Scenario 4 & 0.886 & 1 & 0.805 & 2 \\
Scenario 5 & 0.940 & 1 & 0.870 & 5 \\
Scenario 6 & 0.525 & 1 & 0.310 & 1 \\
Scenario 7 & 0.835 & 0 & 0.682 & \\
\hline
\end{tabular}

the communication data sequences from the laboratorybased experiment found that up to $98 \%$ of sequences had at least one invalid communication event. On average, approximately $2-3 \%$ of sequences were contaminated with invalid communications. The percentage of invalid communications doubled to approximately 4-5\% when a VAD filter was combined with the duration threshold. Similar results were found when applying the same filters to the communication data from the ABM simulation study. The analysis of the laboratory-based communication sequences also showed that longer sequences tended to have a greater number of invalid communications, with the combination of duration threshold and VAD filtering producing a greater number of invalid communications than those filtered using a duration threshold alone. Overall, the results of both studies showed that filters that combined a duration threshold and VAD algorithm detected more invalid communications than those detected when using a duration threshold.

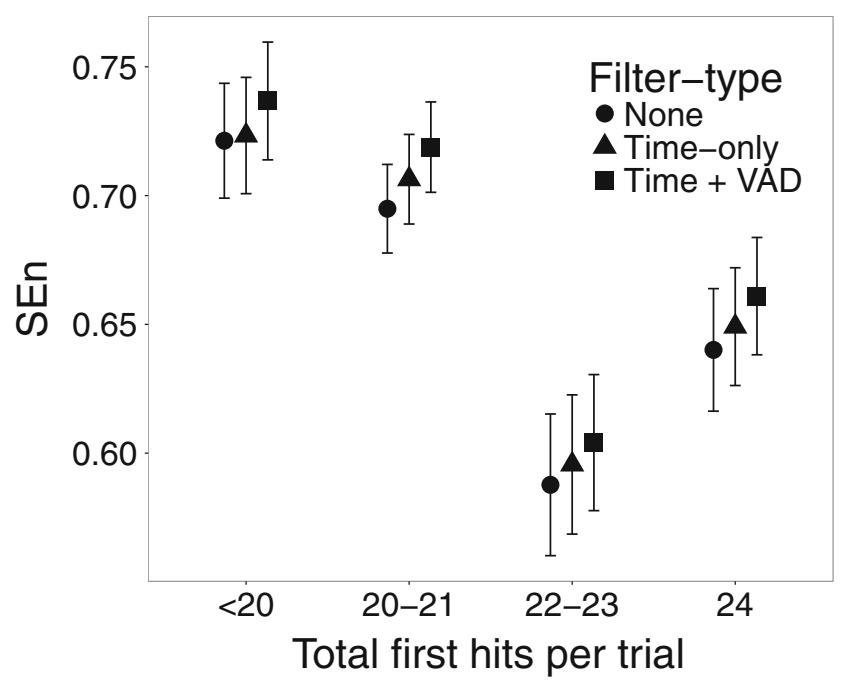

Fig. 9 SEn values as a function of the type of filter used and the total number of hits on first attempt in each trial. Error bars are one standard error of the mean
The second aim of this study was to investigate what the effect of filtering invalid communications had on the complexity of the communication sequences relative to unfiltered data. In the laboratory-based study, we showed that duration threshold filtering produced smaller differences in complexity than those that occurred when combined with VAD. The reductions in complexity that occurred due to filtering became larger as a function of decreasing unfiltered sequence lengths, and this result is consistent with previous research showing that SEn values for larger data sets are more robust to low-level noise and artifacts in the data than that for smaller data sets (Zhang \& Roy, 2001). Furthermore, we found that this pattern of results occurred when comparing sequences of unfiltered data that were trimmed to the length of each matched filtered sequence, and when all communication sequences were trimmed to the same minimum overall length, indicating the effects of filtering on complexity is not due to differences in sequence lengths.

Duration-threshold filtering of communication sequences could potentially be considered a parsimonious method for identifying and removing invalid communications. However, we have shown that duration-threshold filtering alone is not sufficient for this task. A combination of durationthreshold and VAD filtering identified and removed a greater number of invalid communications, and produced larger changes in the complexity of the communication data, with the complexity of sequences using duration threshold and VAD filtering being less correlated with unfiltered sequences. However, duration threshold and VAD filtering did not produce consistent effects on the complexity of the data relative to unfiltered communication sequences, and therefore it was not possible to determine whether filtering data for invalid communications adds complexity (by removing deterministic signals from the data) or reduces complexity (by eliminating noisy signals). The inconsistent effects of filtering were most clearly demonstrated when inspecting how complexity values changed from being significantly different to the mean complexity of surrogate distributions to not significant, and vice versa. Overall, 
the duration threshold filter combined with VAD produced more reversals in statistical difference to surrogate distributions than those for the duration threshold filter alone. However, for the laboratory-based communication data, there was an asymmetry in the findings such that it was more frequently the case that filtering appeared to add complexity (reduce regularity) to the data and the complexity of sequences became less likely to be different to that expected by chance. In contrast, filtering communication sequences from the ABM simulation appeared to produce asymmetrical results, but these asymmetries changed direction over different simulation trials, such that overall differences were minimal.

The third aim of this study was to examine whether the changes in the complexity of communication sequences that occurred due to filtering of invalid communication events had any influence on the relationship between complexity and task performance. The results showed that for our performance data, the relationship between the total number of hits per trial and the SEn of communication sequences was not influenced by the type of filtering used. However, we argue that even though such an effect was not manifest in our analyses, it remains plausible that there is potential for such an effect to occur with other task performance measures that were designed to be more sensitive to the changes in complexity that we found. Furthermore, the relationship between team communication complexity and different phases of communication, where peaks in time series of windowed complexity data have been shown to be associated with phase-shifts in communication content (Wiltshire et al., 2018) and disruptive environmental events (Strang et al., 2012), could be influenced by filtering. This would be especially so if those peaks were based on some threshold change in complexity based on confidence intervals or change-point detection. We base this claim on our finding that there were many occasions in which there were reversals in sample entropy from being significantly different to the 1 st percentile of surrogate distributions and vice versa. On this basis, it is plausible that without filtering of invalid communication events, some entropy time-series peaks could potentially give false alarms of event shifts (false positives), or alternatively, that some event shifts could be missed (false negatives). While we did not have data suitable for such analysis due to sequences being either too short (i.e., in the laboratory-based analysis) or having insufficient events (i.e., in the mission-based analysis), we intend to investigate this hypothesis in future research.

The relationship between sample entropy on task performance that we found provides new information about how the complexity of communication between team members varies depending on the success of their performance. Previous research has been inconsistent regarding the direction of the relationship between complexity and team performance. While some studies suggest that relatively higher complexity is associated with better team performance (e.g., Gorman et al., 2016; Stevens et al., 2012), others have found the reverse; that is, that relatively lower complexity is associated with better team performance (e.g., Wiltshire et al., 2018). Our results showed that as task performance improved (i.e., an increase in the number of successful first hits), the complexity of communication sequences decreased. This is consistent with the findings of Wiltshire et al. (2018) and supports a proposed two-phase model of coordination processes in which 'adaptation' processes are driven by errors (c.f., Marks et al., 2001; Ishak \& Ballard, 2012). That is, with fewer errors there is less need for re-planning/realigning within the team and therefore less complexity in communications. However, the reduction in complexity as a function of improving task performance was not monotonic. Rather, there was a significant increase in communication complexity for teams that achieved perfect performance (i.e., no missed targets in a trial), resulting in a U-shaped function. This upward swing produced a U-curve in the complexity function and is consistent with 'freezing-and-freeing' of degrees of freedom as one gains expertise on a task (Bernstein, 1996) and the notion of 'optimal complexity' which Stergiou et al. (2006) propose to be located somewhere between the upper and lower limits of complexity on a given task.

It should be noted that the two studies from which communication data were reported here were very different in their design and methodology, such that their results cannot be directly compared. Therefore, it was interesting to find that both studies produced similar proportions of invalid communications for both filter types. Given that the two tasks were designed to facilitate a suitable amount of workload relative to the experience and skills of the operators, it might be expected that both studies would produce invalid communications at similar frequencies. It follows, then, that it may be possible that different proportions of invalid communications would be produced as a function of varying workload. A test of this hypothesis would be useful if invalid communications could be shown to be a predictor of operator workload.

We have provided a method for filtering communication sequences that enables discrete communication events to be checked for their validity based on both the duration of the communication event and a voice activity detection algorithm. Although we employed this technique specifically for the filtering of DIS PDUs obtained from communication between team members using a push-totalk system, any audio recordings of communications that have been discretized could benefit from the filtering techniques we have described. The VAD algorithm will take other audio file formats as inputs (such as those produced 
using the Audacity software package; e.g., see Gontar et al., 2017) and determine the presence of voice content. Audio recordings that are intended to be transcribed and analyzed for semantic content and categorization would also benefit from pre-filtering such that invalid communications are ignored, therefore increasing the efficiency of the transcription process that can be time-consuming and expensive to carry out.

In summary, while we found that the effects of filtering on sample entropy did not have an influence on the relationship between complexity and task performance for our particular experimental design, we did find significant changes in complexity when invalid communication events were filtered. Furthermore, we found that filtering of communication data changed the temporal structure of the communication sequences such that comparisons to surrogate distributions resulted in inconsistent significance tests and therefore unpredictable effects. Taken together, the results of this study lead to the conclusion that, as in other domains that analyze time-series or discrete sequences of data (e.g., eye movement and heart-rate data), pre-filtering of invalid communication data should be included during the data cleaning stage of statistical analysis. This is as a matter of good scientific practice, and should help ensure that any inferences made about the relationship between the complexity of communication between team members and their task performance are not confounded by the presence of invalid communication events.

Publisher's Note Springer Nature remains neutral with regard to jurisdictional claims in published maps and institutional affiliations.

\section{References}

Bernstein, N. A. (1996). Resources for ecological psychology. Dexterity and its development. In Latash, M. L., \& Turvey, M. T. (Eds.) Hillsdale: Lawrence Erlbaum Associates, Inc.

Bessette, B., Salami, R., Lefebvre, R., et al. (2002). The adaptive multirate wideband speech codec (AMR-WB). IEEE Transactions on Speech and Audio Processing, 10, 620-636. https://doi.org/10.1109/TSA.2002.804299

Coco, M. I., \& Dale, R. (2014). Cross-recurrence quantification analysis of categorical and continuous time series: An R package. Frontiers in psychology, 5, 510. https://doi.org/10.3389/fpsyg.2014. 00510

Cooke, N. J., Duchon, A., Gorman, J. C., Keyton, J., \& Miller, A. (2012a). Preface to the special section on methods for the analysis of communication. Human Factors, 54, 485-488. https://doi.org/10.1177/0018720812448673

Cooke, N. J., Gorman, J. C., Myers, C., \& Duran, J. (2012b). Theoretical underpinnings of interactive team cognition. In Salas, E., Fiore, S. M., \& Letsky, M. P. (Eds.) New York: Routledge.

Cooke, N. J., Gorman, J. C., Myers, C. W., \& Duran, J. L. (2013). Interactive team cognition. Cognitive science, 37, 255-285.
Francis, C., Best, C., \& Yildiz, J. (2015). Improving air force operator performance through synthetic mission rehearsal. In Proceedings of SimTecT 2015

Gontar, P., Fischer, U., \& Bengler, K. (2017). Methods to evaluate pilots' cockpit communication: Cross-recurrence analyses vs. speech act-based analyses. Journal of Cognitive Engineering and Decision Making, 11(4), 337-352.

Gorman, J. C., Cooke, N. J., Amazeen, P. G., \& Fouse, S. (2012). Measuring patterns in team interaction sequences using a discrete recurrence approach. Human Factors, 54, 503-517.

Gorman, J. C., Martin, M. J., Dunbar, T. A., Stevens, R. H., Galloway, T. L., Amazeen, P. G., \& Likens, A. D. (2016). Cross-level effects between neurophysiology and communication during team training. Human factors, 58, 181-199. https://doi.org/10.1177/ 0018720815602575

Guastello, S. J. (1998a). Creative problem solving groups at the edge of chaos. The Journal of Creative Behavior, 32(1), 38-57.

Guastello, S. J., Hyde, T., \& Odak, M. (1998b). Symbolic dynamic patterns of verbal exchange in a creative problem solving group. Nonlinear Dynamics. Psychology, and Life Sciences, 2(1), 35-58.

Guastello, S. J. (2000). Symbolic dynamic patterns of written exchanges: Hierarchical structures in an electronic problem solving group. Nonlinear Dynamics, Psychology, and Life Sciences, 4(2), 169-187.

Holm, S. (1979). A simple sequentially rejective multiple test procedure. Scandinavian Journal of Statistics, 6, 65-70.

Ishak, A. W., \& Ballard, D. I. (2012). Time to re-group: A typology and nested phase model for action teams. Small Group Research, 43, 3-29.

Kozlowski, S. W., \& Ilgen, D. R. (2006). Enhancing the effectiveness of work groups and teams. Psychological science in the public interest, 7, 77-124.

MacMillan, J., Entin, E. B., Hess, K. P., \& Paley, M. J. (2004). Measuring performance in a scaled world: Lessons learned from the distributed dynamic decision making (DDD) synthetic team task. Scaled Worlds: Development, Validation, and Applications. Ashgate Publishing Co.

Marks, M. A., Mathieu, J. E., \& Zaccaro, S. J. (2001). A temporally based framework and taxonomy of team processes. The Academy of Management Review, 23, 356-376.

Murase, T., Poole, M. S., Asencio, R., \& McDonald, J. (2017). Sequential synchronization analysis. In Group processes (pp. 119144). Springer.

Parker, J., Best, C. J., Funke, G., Strang, A., \& Marion, K. (2016). An investigation of coding schemes for sample entropy analysis of communications data. In HFES 2016 : Proceedings of the 56th Human Factors and Ergonomics Society Annual Meeting (pp. 111-115)

Richman, J. S., \& Moorman, J. R. (2000). Physiological time-series analysis using approximate entropy and sample entropy. American Journal of Physiology-Heart and Circulatory Physiology, 278, H2039-H2049.

Russell, S. M., Funke, G. J., Knott, B. A., \& Strang, A. J. (2012). Recurrence quantification analysis used to assess team communication in simulated air battle management. In HFES 2012 : Proceedings of the 56th Human Factors and Ergonomics Society Annual Meeting (pp. 468-472).

Sokunbi, M. O. (2014). Sample entropy reveals high discriminative power between young and elderly adults in short fMRI data sets. Frontiers in Neuroinformatics, 8, 1-12. https://doi.org/10.3389/ fninf.2014.00069

Stergiou, N., Harbourne, R., \& Cavanaugh, J. (2006). Optimal movement variability: A new theoretical perspective for neurologic 
physical therapy. Journal of Neurologic Physical Therapy, 30, 120-129.

Stevens, R. H., Galloway, T. L., Wang, P., \& Berka, C. (2012). Cognitive neurophysiologic synchronies: what can they contribute to the study of teamwork? Human Factors, 54, 489-502. https://doi.org/10.1177/0018720811427296

Strang, A. J., Horwood, S., Best, C. J., Funke, G. J., Knott, B. A., \& Russell, S. M. (2012). Examining temporal regularity in categorical team communication using sample entropy. In HFES 2012 : Proceedings of the 56th Human Factors and Ergonomics Society Annual Meeting.

Watkinson, P., Best, C., Von Trevor, K., \& Jia, D. (2015). An investigation of the directional relationship between cohesiveness and action team performance. In Proceedings of the Australian Psychological Society Industrial and Organisational Psychology conference.
Weippert, M., Behrens, M., Rieger, A., \& Behrens, K. (2014). Sample entropy and traditional measures of heart rate dynamics reveal different modes of cardiovascular control during low intensity exercise. Entropy, 16, 5698-5711.

Wiltshire, T. J., Butner, J. E., \& Fiore, S. M. (2018). Problem-solving phase transitions during team collaboration. Cognitive Science, 42(1), 129-167.

Yentes, J. M., Hunt, N., Schmid, K. K., Kaipust, J. P., McGrath, D., \& Stergiou, N. (2013). The appropriate use of approximate entropy and sample entropy with short data sets. Annals of Biomedical Engineering, 41, 349-365. https://doi.org/10.1007/s10439-0120668-3

Zhang, X. S., \& Roy, R. J. (2001). Derived fuzzy knowledge model for estimating the depth of anesthesia. IEEE Transactions on Biomedical Engineering, 48, 312-323. 